INPLASY

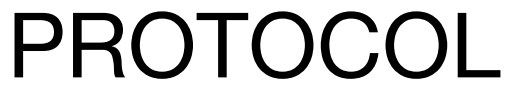

To cite: Lan et al. Integrated Traditional Chinese and Western Medicine for ulcerative colitis with diabetes: A protocol for systematic review and meta-analysis. Inplasy protocol 2020120087. doi:

10.37766/inplasy2020.12.0087

Received: 16 December 2020

Published: 16 December 2020

Corresponding author:

Yuze Lan

lanyzself@163.com

Author Affiliation:

Gansu University of Chinese Medicine

Support: Yes.

Review Stage at time of this submission: The review has not yet started.

Conflicts of interest: None.

\section{Integrated Traditional Chinese and Western Medicine for ulcerative colitis with diabetes: A protocol for systematic review and meta-analysis}

Lan, Y1; Bai, Y2; Zhu, X³.

Review question / Objective: This study aimed to access the effectiveness and safely of integrated Traditional Chinese and Western Medicine for ulcerative colitis with diabetes through the way of systematic review and meta-analysis.

Condition being studied: Ulcerative colitis with diabetes.

Information sources: We plan to search eight electronic databases (four English databases: PubMed, Embase, Cochrane Library and Web of Science; four Chinese databases: China biology Medicine (CBM), China National Knowledge Infrastructure (CNKI), Wanfang Data and Chinese Scientific Journal Database (VIP)) to identify qualifying studies published from database inception until 1 December 2020. The search strategy will be formulated by library search specialist and epidemiologist. The Medical Subject Headings (MeSH) and free words will be used to search in the above databases. Boolean Logic (AND, OR) will be combined using in the search terms when needed. Human studies and peerreviewed journal articles published in English or Chinese will be restrict in our search. The search strategies for PubMed can be found in Table 1. In addition, we will manually search the reference lists of all included studies for any further potentially relevant studies.

INPLASY registration number: This protocol was registered with the International Platform of Registered Systematic Review and Meta-Analysis Protocols (INPLASY) on 16 December 2020 and was last updated on 16 December 2020 (registration number INPLASY2020120087).

\section{INTRODUCTION}

Review question / Objective: This study aimed to access the effectiveness and safely of integrated Traditional Chinese and Western Medicine for ulcerative colitis with diabetes through the way of systematic review and meta-analysis.

Condition being studied: We plan to search eight electronic databases (four English databases: PubMed, Embase, Cochrane 
Library and Web of Science; four Chinese databases: China biology Medicine (CBM), China National Knowledge Infrastructure (CNKI), Wanfang Data and Chinese Scientific Journal Database (VIP)) to identify qualifying studies published from database inception until 1 December 2020. The search strategy will be formulated by library search specialist and epidemiologist. The Medical Subject Headings (MeSH) and free words will be used to search in the above databases. Boolean Logic (AND, OR) will be combined using in the search terms when needed. Human studies and peer-reviewed journal articles published in English or Chinese will be restrict in our search. The search strategies for PubMed can be found in Table 1. In addition, we will manually search the reference lists of all included studies for any further potentially relevant studies.

\section{METHODS}

Participant or population: All participants are diagnosed with UC by medical history, endoscopic parameters, clinical evaluation and histopathology. And infectious colitis will be excluded careful. In addition, patients with UC also suffer from diabetes (included type 1 diabetes and type 2 diabetes). The diagnostic criteria for diabetes are typical symptoms of diabetes (polydipsia, polyuria, polyphagia, and weight loss) plus random plasma glucose $\geq 11.1 \mathrm{mmol} / \mathrm{L}$ or fasting plasma glucose (FPG) $\geq 7.0 \mathrm{mmol} / \mathrm{L}$ or oral glucose tolerance test (OGTT) 2-Hour plasma glucose $(2 \mathrm{hPG}) \geq 11.1 \mathrm{mmol} / \mathrm{L}$. All patients must be adults ( $\geq 18$ years). There will be regardless about gender, region, education, economic, and other factor.

Intervention: The control group received conventional treatment of Western medicine, and did not restrict the type of drugs, dosage form, frequency, and course of treatment. The intervention measures of the experimental group were combined with Traditional Chinese Medicine on the basis of the control group, included Chinese herbal medicine and Chinese patent medicine. Other intervention measures of Traditional Chinese Medicine will be ruled out, such as acupuncture, moxibustion, massage.

Comparator: The control group received conventional treatment of Western medicine, and did not restrict the type of drugs, dosage form, frequency, and course of treatment.

Study designs to be included: Randomized controlled trials (RCTs) will be included in this review. Self-controlled studies, nonRCTs, randomized crossover studies, quasi-randomized trials, case report, case series and cross-sectional studies will be excluded.

Eligibility criteria: Type of studies: Randomized controlled trials (RCTs) will be included in this review. Self-controlled studies, non-RCTs, randomized crossover studies, quasi-randomized trials, case report, case series and cross-sectional studies will be excluded. Type of participants: All participants are diagnosed with UC by medical history, endoscopic parameters, clinical evaluation and histopathology. And infectious colitis will be excluded careful. In addition, patients with UC also suffer from diabetes (included type 1 diabetes and type 2 diabetes). The diagnostic criteria for diabetes are typical symptoms of diabetes (polydipsia, polyuria, polyphagia, and weight loss) plus random plasma glucose $\geq 11.1 \mathrm{mmol} / \mathrm{L}$ or fasting plasma glucose (FPG) $\geq 7.0 \mathrm{mmol} / \mathrm{L}$ or oral glucose tolerance test (OGTT) 2-Hour plasma glucose $(2 \mathrm{hPG}) \geq 11.1 \mathrm{mmol} / \mathrm{L}$. All patients must be adults ( $\geq 18$ years). There will be regardless about gender, region, education, economic, and other factor. Type of interventions: The control group received conventional treatment of Western medicine, and did not restrict the type of drugs, dosage form, frequency, and course of treatment. The intervention measures of the experimental group were combined with Traditional Chinese Medicine on the basis of the control group, included Chinese herbal medicine and Chinese patent medicine. Other intervention measures of Traditional Chinese Medicine will be ruled out, such as acupuncture, moxibustion, massage. 
Information sources: We plan to search eight electronic databases (four English databases: PubMed, Embase, Cochrane Library and Web of Science; four Chinese databases: China biology Medicine (CBM), China National Knowledge Infrastructure (CNKI), Wanfang Data and Chinese Scientific Journal Database (VIP)) to identify qualifying studies published from database inception until 1 December 2020. The search strategy will be formulated by library search specialist and epidemiologist. The Medical Subject Headings (MeSH) and free words will be used to search in the above databases. Boolean Logic (AND, OR) will be combined using in the search terms when needed. Human studies and peer-reviewed journal articles published in English or Chinese will be restrict in our search. The search strategies for PubMed can be found in Table 1. In addition, we will manually search the reference lists of all included studies for any further potentially relevant studies.

Main outcome(s): Total effective rate (referring to the diagnostic criteria of ulcerative colitis in the Consensus Opinions on Diagnosis and Treatment Standards of Inflammatory Bowel Disease in China). (Total effective rate $=$ number of complete remission + effective number)/ total number $* 100 \%$. Complete remission is defined as the clinical symptoms is disappearance, and the mucosa is found to be roughly normal by colonoscopy reexamination. Effective represents the basic disappearance of clinical symptoms, mild inflammation of mucosa or pseudopolyp formation by colonoscopy reexamination. Ineffective represents no improvement of clinical symptoms, endoscopy, and pathological examination results after treatment.

Additional outcome(s): Recurrence rate, symptom score, colonoscopic score, serum bloody test (such as Antineutrophil cytoplasmic antibodies, ANCA), mucosal healing rate and incidence of adverse reactions.
Data management: A pre-developed standardized data collection form will be used to extracted from all eligible studies. The relevant information including title, first author, publication year, sample size, average age, sex ratio, average course of disease, drug type, dosage form, frequency, course of treatment, and outcome indicators. In addition, important citations, funding agencies and potential conflicts of interest will also be collected. We will contact the corresponding author by email if the required data are missing or unclear. Data will be independently extracted by two reviewers. There will be discussed between two reviewers if discrepancies arise. If necessary, a third reviewer will be consulted to achieve consensus.

Quality assessment / Risk of bias analysis: The quality of all included studies will be independently assessed by two reviewers, and any disagreements will be determined by the third author. For the RCTs, the quality will be assessed by Cochrane Risk of bias tool. This tool consists of the following seven questions: random sequence generation (selection bias), allocation concealment (selection bias), blinding of participants, blinding of outcome assessment (performance bias), incomplete outcome data (attrition bias), selective reporting (reporting bias) and other bias ("other bias" is determined by consensus of the investigators). Each question has three answers: Yes' (low risk of bias), 'No' (high risk of bias) or 'Unclear' (lack of information or uncertainty over the potential bias).

Strategy of data synthesis: If possible, meta-analysis will be undertaken for each of the outcomes. For continuous variable data, we will used mean differences (MDs) with $95 \%$ Cls as summary statistics. If studies had used different measurement instruments or units to measure an outcome, we plan to use the standardized mean difference (SMD). For dichotomous variable data, we will calculate MantelHaenszel Odds Ratio (OR) with $95 \%$ Cls as summary statistics from the numbers of events in control and intervention groups. 
We will consider a result to be statistically significant if $P<0.05$. If outcomes cannot be meta-analyzed, we will performer a descriptive analysis. The RevMan 5.3 software developed by the Cochrane Collaboration will be used for all statistical analysis.

Subgroup analysis: If necessary or possible, results will be analyzed for the following subgroups: (1) type of diabetes: type 1 diabetes, type 2 diabetes; (2) age groups: 18-45 years, 46-60 years, 61-75 years, > 75 years; (3) sex: man, woman.

Sensibility analysis: We will explore clinical and statistical sources of heterogeneity among the different groups of RCTs by $\mathbf{I}^{2}$ statistics and $X^{2}$ test ( $50 \%$ deemed high). If heterogeneity is moderate, we will use random effects model to analysis. If heterogeneity is high, we will perform a subgroup analysis. We also will performer a descriptive analysis if cannot perform subgroup analysis.

Language: English and Chinese.

Country(ies) involved: China.

Keywords: ulcerative colitis; diabetes; integrated traditional chinese and western medicine; systematic review; metaanalysis.

Contributions of each author:

Author 1 - Yuze Lan.

Email: lanyzself@163.com

Author 2 - Yali Bai.

Author 3 - Xiangdong Zhu.

Email: zhuxiangdong33@163.com 